\title{
Smelling objects
}

\author{
Becky Millar ${ }^{1}$
}

Received: 13 February 2017 / Accepted: 13 December 2017 / Published online: 20 December 2017

(C) The Author(s) 2017. This article is an open access publication

\begin{abstract}
Objects are central to perception and our interactions with the world. We perceive the world as parsed into discrete entities that instantiate particular properties, and these items capture our attention and shape how we interact with the environment. Recently there has been some debate about whether the sense of smell allows us to perceive odours as discrete objects, with some suggesting that olfaction is aspatial and doesn't allow for object-individuation. This paper offers two empirically tractable criteria for assessing whether particular objects are exhibited in perceptual experience-(1) susceptibility to figure-ground segregation and (2) perceptual constancies - and argues that these criteria are fulfilled by olfactory perception, and thus there are olfactory objects. I argue that there are, in fact, two different ways that olfaction allows for figure-ground segregation. First, I look at various Gestalt grouping principles, which are thought to govern when features are perceived as grouped into structured wholes, segregated from everything around them. I argue that these principles apply to olfactory experience, providing evidence of non-spatial figure-ground segregation. Second, I defend the contentious idea that a spatial variety of figureground segregation can also occur in olfaction. To see this, however, we need to look to empirical evidence showing that tactile stimulation and bodily movements play a crucial role in olfactory phenomenology. Finally, I draw on empirical evidence and olfactory phenomenology to argue that there are perceptual constancies in olfactory experience, allowing us to perceive odours as coherent objects that survive shifts in our perspectives on the world.
\end{abstract}

Keywords Perception · Olfaction · Objects · Figure-ground · Constancies

$\triangle \quad$ Becky Millar

rmillar2@exseed.ed.ac.uk

1 C/O 2.16, Postgraduate Office, Dugald Stewart Building, The University of Edinburgh, 3 Charles Street, Edinburgh EH8 9AD, UK 


\section{Introduction}

Objects are central to perception and our interactions with the world. We perceive the world as parsed into discrete entities that instantiate properties, and these items capture our attention and shape how we interact with the environment. For example, our visual experience divides the world up into clearly distinguished, meaningful units like flowers, dogs and chairs. The objects we perceive are key to our understanding of the world; concepts, beliefs and desires relating to objects are ubiquitous. However, to understand the nature of these entities, we need to uncover the criteria for perceptual objecthood. What conditions must be fulfilled for something we perceive to be an object, as opposed to, say, a range of nebulous, unbounded properties? And could the sense of smell allow the perception of discrete objects?

Philosophers have claimed that compared to visual perceptions, olfactory experiences are "smudgy" (e.g. Batty 2010a) and do not involve the perception of discrete objects. Rather, they only allow for the perception of either nebulous olfactory properties (Matthen 2005) or objects in a very weak sense. These weaker objectbased approaches include a non-phenomenological variety of objecthood from Lycan (1996, 2000) and an existentially-quantified variety from Batty (2010a,b, 2011, 2014a,b), whereby all olfaction allows us to perceive is that there is an undifferentiated something-or-other in one's vicinity that instantiates properties (Batty 2010a). This paper argues against such views in favour of a robust kind of olfactory objecthood. We experience odours as discrete units that bear properties and retain their identities through perspectival change. I argue that figure-ground segregation (which underpins object-individuation) and perceptual constancies (which, I claim, underpin object-recognition) are individually necessary and jointly sufficient for this type of objecthood. Paying attention to Gestalt psychology, the role of bodily movement and tactile stimulation in olfaction, and olfactory phenomenology, I argue that these criteria apply to the olfactory case.

The structure of this paper is as follows. Section 1 provides background on the debates surrounding the issue of perceptual objecthood. Section 2 offers two empirically tractable criteria for assessing whether discrete perceptual objects are exhibited in a given perception-susceptibility to figure-ground segregation and perceptual constancies. While the importance of these aspects of object perception has been highlighted elsewhere, I offer a novel argument for the claim that they are individually necessary and jointly sufficient for objecthood. In Sect. 3, I argue that both figureground segregation and perceptual constancies are exhibited in olfactory perception. First, in Sect. 3.1, I argue in favour of a kind of non-spatial figure-ground segregation in smell. Drawing analogies with the other senses, I suggest that the Gestalt grouping principles - thought to govern how features are grouped together and segregated from everything else within the perceptual field—can provide valuable evidence for a non-spatial form of figure-ground segregation in olfaction. In Sect. 3.2, I argue that contrary to majority opinion, that there can also be spatial olfactory figure-ground segregation. To see this, we need to look to empirical evidence showing that tactile stimulation and bodily movements play a crucial role in olfactory phenomenology. Finally, in Sect. 3.3, I highlight a number of perceptual constancies exhibited in olfactory experience, considering both olfactory phenomenology and empirical research. 


\section{Background}

The notion of perceptual objecthood that I will be primarily concerned with in this paper is a robust phenomenological sense of objecthood: single, discrete entities exhibited in our experience that nonetheless exhibit many distinct properties. For example, in the case of vision, when we perceive a flower, we perceive it as a single object, despite it consisting of a long green stem, petals of different colours and so forth. This relies on our capacity for object individuation-perceptual systems can discern that groups of features belong to the same individual. Another crucial aspect of perceptual objecthood is that we perceive objects as remaining constant amid changes in our own perspective, movements of the objects themselves and, within limits, as they change over time. As Rüdiger von der Heydt says in a recent review article, "One characteristic of perceptual objects is continuity (object permanence). When an object is briefly occluded by a foreground object and then reappears, it is perceived as the same object. A token seems to persist" (2015, p. 6). Likewise, O'Callaghan says: “objects perceptibly persist and survive changes" (2016, p. 1273). This apparent persistence of an object is closely connected with our object recognition abilities (see Sect. 2.2 for discussion of the connection between perceptual constancies and object recognition), which are considered important aspects of object perception. Object recognition encompasses abilities such as tracking objects, perceiving them as complete objects despite occlusions or the limitations of our particular perspective (amodal completion), and perceiving them as persisting through time.

There are interesting regularities between the entities we perceive as objects and in the particular processes involved in perceiving objects. For example, the Gestalt psychologists uncovered an array of principles that appear to govern when we perceive worldly features as grouped together. Some examples of this include: the 'law of common fate', which states that we tend to group together elements that move together; the laws of similarity and proximity, which say that features perceived as similar and/or in close proximity are more likely to be perceived as grouped together into objects; the law of good form, which says that we tend to group together elements that are coherent, balanced, simple and so forth; and the law of closure, which says that features are more likely to be perceived as grouped if they are part of a closed figure (Wertheimer 1923/1938). Researchers have found that many of these regularities apply not only visual perception but also perceptions via other senses, such as audition (e.g. Bregman 1990). This motivates some of the discussion of perceptual objecthood because there are significant similarities in how we perceive and parse the world via our different senses, suggesting that there may be a notion of objecthood applicable to non-visual perception. Here I aim to find non-visuocentric necessary and sufficient conditions for a robust notion of perceptual objecthood. The Gestalt principles-as formulated by Wertheimer-are not themselves suitable for this, because they are defeasible (involving a ceteris paribus clause) and seem to be neither necessary nor sufficient for object-perception. However, as we shall see in Sect. 3, they still provide strong evidence of figure-ground segregation.

Although a number of researchers have considered whether we perceive objects through non-visual modalities such as audition (e.g. Kubovy and Van Valkenburg 2001; Griffiths and Warren 2004; Matthen 2010), taste (Stevenson 2014), and smell 
(for detailed discussions see Lycan 1996; Batty 2010a, 2011, 2014a, b; Carvalho 2014; Young 2016), ${ }^{1}$ most discussions of objecthood still centre on the visual case, which is often taken to be paradigmatic of object perception. Perhaps this is in part because in the visual case these perceived objects are mainly ordinary, three-dimensional objects. When we look at a red ball, rather than merely seeing features such as redness and roundness, we can perceive these features as belonging to an object that is red and round. Yet even in the visual case, perceptual objects are a broader category than everyday objects like balls and flowers. O'Callaghan (2016, p. 1274) points out that we see items such as shadows and rainbows in an object-like way even though they aren't ordinary material objects. We still see such items as being, like the ball, spatially-arrayed in our visual field and as exhibiting (relatively) clear boundaries, and as persisting over time and as we move around. Thus, an adequate phenomenological account of objecthood ought to allow for such cases.

The challenge of providing a satisfactory account of objecthood is even more pronounced when considering the sense of smell. In contrast to the visual case, it is controversial whether we perceive olfactory objects at all. Our olfactory perceptions are unlike our visual perceptions in that they don't seem to present us with items with clear, sharply distinguished edges. Thus, it is not immediately clear whether olfaction could allow the perception of discrete objects, and as we shall see, there is little consensus on this issue.

Moreover, there are a number of potential complications in arguing in favour of olfactory objecthood. For example, an initial problem is to decide what sort of objects we could perceive via olfaction. There are two main candidates for olfactory objecthood: the distal object (e.g. the rose) or the volatile chemicals/odour (e.g. the rose odour). There are good reasons, discussed in detail elsewhere (e.g. see Lycan 1996; Batty 2010a; Richardson 2013; Young 2016) to suspect that we do not directly perceive the rose itself through olfaction. We can smell a rose scent without having to be in the direct vicinity of a rose, and a rose scent may persist after the rose itself is removed or destroyed. It is also possible to give rise to a rose scent artificially-say, through a rose-scented air freshener. It would be strange to say that our perceptions in such cases are non-veridical because of the lack of an actual rose. Thus, the most common view among those who endorse an object-based account is the immediate objects that we perceive through olfaction are odours, rather than source objects. It is sometimes added that we can still indirectly perceive the source objects via the perception of the odour (Lycan 1996; see also Cavedon-Taylor forthcoming for a review of these issues). While the issue of whether source objects are represented in olfactory perception is important in determining the intentional objects of olfaction, here my concern is whether we immediately perceive odours as objects in a phenomenological sense (which will be spelled out in Sect. 2). My claim is that for odours to count as objects in this sense, they must be perceived as discrete entities, which instantiate properties and survive shifts in perspective. I remain neutral on the question of whether olfaction may also involve an indirect representation of source objects, and shall not discuss these issues further as they are orthogonal to the issues at hand. For the purposes of

\footnotetext{
1 See also briefer remarks on such issues from Peacocke (1983) and Matthen (2005).
} 
this paper I shall assume that if we immediately perceive objects through olfaction, these objects are odours. This assumption is shared in the key literature that I discuss throughout this paper (e.g. Lycan 1996, 2000; Wilson and Stevenson 2003, 2006, 2007; Batty 2010a, 2011, 2014a, b; Carvalho 2014).

A second, related complication is that even if we agree that it is odours rather than source objects that we directly perceive through olfaction, there is some debate on the nature of these odours. For the purposes of this paper, I will follow philosophers such as Lycan (1996) and Roberts (2015) in taking an odour to be a molecular cloud of volatile particles, which may instantiate olfactory properties such as smokiness, sweetness, and so forth. I shall not discuss the nature of odours further in this paper (see Cavedon-Taylor forthcoming for an in-depth discussion), but it is worth noting that not all parties in the debates about olfaction share the same idea of what an odour is.

The theorists that I shall discuss in detail in this paper all endorse world-directed accounts of olfaction. However, it is worth noting that this is itself somewhat controversial and some philosophers have claimed that our olfactory experiences may be merely sensational; i.e. olfaction only makes us aware of properties of our own experiences rather than properties of, or objects in, the world. The clearest example of this comes from a brief but suggestive remark from Peacocke: "a sensation of... [smell] may have no representational content of any sort, though of course the sensation will be of a distinctive kind" (1983, p. 5). Others have claimed that the phenomenology of olfaction is in accordance with the sensation-based view, even if the sensation-based view is inaccurate for other reasons (e.g. Lycan 1996). With a sensation-based view, we do not perceive objects or worldly features in the case of olfaction; there are in fact no worldly entities to which we attend when we smell, as sensations are non-world directed. Most researchers, however, seem keen to accommodate olfaction in worlddirected accounts of perception, rejecting the sensation-based view. ${ }^{2}$ There have been a number of different world-directed approaches to olfaction.

The first world-directed approach to smell is the feature-based view. Those that endorse this approach concede that olfaction is world-directed, but claim that what we olfactorily perceive are not objects but only properties or features (see Matthen's brief discussion of olfaction in 2005, pp. 284-285 for such an account). We don't smell the fruitiness, smokiness and maltiness of a coffee as bound together, but instead simply perceive these properties, free of any particular object. Matthen says that smells, "have, at best, a primitive - that is, an undifferentiating - feature-location structure-every smell of which I am aware is simply here" (p. 284). He adds that olfactory content "does not come in object-attribute form" (ibid.). This sort of feature-based approach has also traditionally been dominant within olfactory science, where the primary goal has been to understand olfaction by identifying how different features of a chemical stimulus are represented in olfactory experience. ${ }^{3}$ However, the tides are turning in

\footnotetext{
2 Here I use the term "world-directed" to refer to any theory of perception that takes worldly entities to be accessible or represented through our experience. This would cover varieties of representationalism and relationalism (such as direct realism). I intend my discussion in this paper to be neutral between these different approaches.

${ }^{3}$ See Wilson and Stevenson (2006) for an in-depth discussion of this model of olfactory experience.
} 
the olfactory sciences and an object-based approach to olfaction, advocated by Wilson and Stevenson (2006, 2007), has recently gained in popularity. Wilson and Stevenson (2006) argue that an object-based approach can make sense of a growing body of data from neurobiology and psychology. In particular, they think an object-based approach better accounts for the behavioural evidence and for the need to perceive biologically salient groups of odorants amid changing olfactory stimulation (p. 1892).

Among the object-based approaches to olfaction, there are wide ranging views. First is the abstract view, endorsed by Batty (2010a, 2011). This view is similar to that of Matthen (2005), but allows for a weak, existentially quantified form of olfactory objecthood. For Batty, olfactory experience only ever involves the representation that there is something-or-other in one's vicinity, instantiating olfactory properties. She argues that we never attribute olfactory properties to particular objects, and there cannot be any object-individuation in the case of olfaction. A coffee smell and a lemon smell perceived concurrently won't be experienced as individuated objects but simply properties instantiated by something or other "here". In this paper, I will argue in favour of a much stronger notion of olfactory objecthood—one in which smelling does allow us to parse the olfactory world into individuated objects. We perceive particular (rather than existentially quantified) olfactory objects.

Lycan $(1996,2000)$ also endorses a weak variety of olfactory objecthood. For Lycan, there are olfactory objects in the sense that our olfactory experience represents odours-and an odour is "a vaporous emanation, a diffusing collection of molecules typically given off from a definite physical source... they are public physical entities available for sensing by anyone who happens, fortunately or unfortunately, by" (1996, p. 91). Like Batty, however, he does not allow for the representation of discrete objects in experience. His account is based upon the idea that taking olfaction to represent odours - public physical entities - allows us to best account for the olfactory correctness conditions discussed above; an experience of a rose smell is veridical just in case there is a rose odour in one's vicinity, regardless of whether there is also a rose nearby. Yet he thinks that this sort of world-directed object perception is not apparent on phenomenological grounds. In fact, he thinks that "phenomenally speaking, a smell is just a modification of our consciousness, a qualitative condition or quale... in us, lingering uselessly in the mind without representing anything" (1996, p. 90). In what follows, I shall argue in favour of a kind of phenomenological objecthood in olfaction-we perceive odour-objects that exhibit figure-ground segregation and perceptual constancies.

Both the feature-based and weaker object-based approaches are largely motivated by the apparent paucity of spatial information associated with olfactory experiences, as compared to other senses. Vision-which is taken to provide the paradigmatic example of object-perception-allows us to differentiate objects and understand their spatial relations with one another and to our own bodies. We see objects as spatially extended and bounded entities, arrayed within the visual field. In contrast, among theorists on all sides of the debate regarding olfactory objects, there is a common suspicion that olfactory perception is aspatial (e.g. Lycan 2000; Wilson and Stevenson 2006) or spatially undifferentiated (Matthen 2005; Batty 2011, 2014b; Carvalho 2014). Carvalho (2014) and Wilson and Stevenson (2006) agree that smell is aspatial, but think that it is possible to perceive individuated objects in a non-spatial manner, while theorists 
such as Batty (2010a,b) and Matthen (2005) treat spatial differentiation as a prerequisite for object-individuation. This idea that spatiality is key to object individuation is quite intuitive given the primacy of vision within perceptual research; in vision, we segregate objects based on spatial locations and their spatially situated edges. Thus, if olfaction fails to be adequately spatially differentiated, this may give prima facie credence to a feature-based or a weak object-based approach (although as we shall see, there are legitimate non-spatial types of figure-ground segregation).

Some such as Carvalho (2014) and Wilson and Stevenson (2003, 2006, 2007) offer stronger accounts of object-perception, taking olfactory experience to present to us particular odour-objects individuated on the basis of their chemical structure. While my own view is more closely aligned with these approaches, both Carvalho and Wilson and Stevenson agree with Batty and Lycan that olfaction does not allow for spatial discrimination of odours, and as we shall see, their arguments for the individuation of odours are alone insufficient to counter Batty's objections. In what follows, I will argue that odours can be individuated both in an aspatial manner based on Gestalt grouping principles and in a spatial manner when we take into account the role of bodily movement and tactile stimulation for olfactory phenomenology.

Finally, there have also been criticisms that target the whole dispute between the different object-based and feature-based approaches. Theorists such as Cooke and Myin (2011) and Barwich (2014) argue in favour of more process-driven approaches, accusing philosophers such as Batty and Lycan of taking the phenomenal character of perceptions to be in some way independent of the processes that give rise to these experiences, a view that Barwich and Cooke and Myin call the Independence Thesis. Allegedly, those guilty of endorsing this thesis tend to assume that sensory perceptions can be understood as static, autonomous units, whose properties may or may not be correlated to the properties of physical objects. Barwich (2014) argues that we can understand olfaction without recourse to perceptual objects or properties of objects, paying attention instead to olfactory processes. I shall not address such views in detail, but will in Sect. 3.2 illustrate how we can take insights from such approaches, while still endorsing an object-based account.

In what follows, I shall oppose the feature-based views and the weaker object-based views of Batty and Lycan, and will argue that there are perceptual objects in olfaction in a robust sense. Olfaction allows the perception of particular objects, rather than merely existentially quantified something-or-others in one's vicinity. I will begin by providing necessary and sufficient conditions for this robust phenomenological sense of objecthood before showing that these criteria are fulfilled in olfactory perception.

\section{Necessary and sufficient conditions for objecthood}

This section argues that figure-ground segregation and perceptual constancies are each necessary for the perception of particular (rather than merely existentially quantified) objects, and that they are jointly sufficient. As mentioned above, discrete objects exhibit boundaries and are able to survive changes in our own perspective, as they move and (to some extent) over time. These phenomenological features of perception involve abilities to discern the boundaries of objects (this falls under the category of object 
individuation) and to recognise objects across different viewing conditions and in different contexts (abilities that are central to object recognition in ways I discuss below in Sect. 2.1). Whether these abilities are accounted for in one's theory of objecthood is a useful test for determining whether we have adequate phenomenological criteria for object perception; the phenomenology of experience ought not be wholly disconnected from the skills involved in perception.

Figure-ground segregation is the defining feature of the ability to perceive boundaries (object individuation), while perceptual constancies allow perceived objects to appear invariant across different perspectives and amid changes (underpinning object recognition abilities). First, I shall look at figure-ground segregation.

\subsection{Figure-ground segregation}

"Figure-ground segregation" refers to our ability to distinguish figures from backgrounds. This figure-ground structure is central to our visual experiences: we perceive, for example, houses against a background of the sky, and books against the background of a desk. We are unable to take in a whole detailed scene at once due to the limitations of our perceptual access to the world, so we attend to a particular figure or set of figures at a given time, relegating everything else to background. We perceive these backgrounds as relatively undifferentiated and less determinate than the figures themselves.

It has been suggested by Kubovy and Van Valkenburg (2001) that susceptibility to figure-ground segregation is the defining feature of perceptual objects; it is both necessary and sufficient for the perception of objects (p. 102). Those features of the world that become figures for us are perceptual objects. A number of other researchers have agreed that figure-ground segregation is central to the perception of particular objects, and have used this criterion to argue both for (e.g. Wilson and Stevenson 2007; Carvalho 2014) and against (e.g. Batty 2011, 2014b) the idea that olfactory experience could allow for objects in this sense. Part of Kubovy and Van Valkenburg's motivation for taking figure-ground separation to be the defining feature of objecthood is to provide a non-visuocentric account of perceptual objects. Research suggests that figure-ground segregation is exhibited in non-visual types of sense perception, such as auditory experience. For example, audition researcher Bregman (1990) claims that hearing involves the parsing of auditory scenes into distinct auditory streams-which can be understood as a kind of boundary allocation — and studies show that listeners are unable to pay attention to more than one sound stream at a time, which is also indicative of figure-ground segregation. One such study by Bregman and Campbell (1971) found that perceivers were unable to judge the order of sounds presented in a repetitive cycle where these sounds were experienced as two distinct streams. Participants were presented with three high-pitched sounds (ABC) and three lowpitched sounds (123) in the order A-1-B-2-C-3. However, the majority of participants experienced the order as either A-B-C-1-2-3 or 1-2-3-A-B-C as they were only able to pay attention to one stream at a time; one stream at a time is experienced as a figure against a background. As I shall discuss in Sect. 3.1, this suggests that figure-ground 
segregation need not be a spatial feature of perception and can be applied to non-visual senses such as audition.

Kubovy and Van Valkenburg are right to avoid a visuocentric notion of perceptual objecthood given the similarities in how we perceive the world through different senses-e.g. both vision and audition involve figure-ground segregation, Gestalt grouping principles, and so forth. Figure-ground segregation is at the heart of 'object-individuation', and a successful phenomenological account of objectperception should highlight the importance of this capacity. Figure-ground segregation allows us to make sense of the idea that perceptual objects are discrete and have boundaries. In fact, figure-ground segregation is both necessary and sufficient for the exhibition in experience of boundaries (which, as suggested by the auditory case above, need not be spatial): if there is figure-ground segregation, there must be boundaries allowing the separation of the figure from background. Likewise, if we perceive something as having boundaries, this entails that we perceive it as segregated from everything else around it. Boundedness and figure-ground segregation are two sides of the same coin. Boundedness (and thus, figure-ground segregation) is certainly necessary for a robust sense perceptual objecthood that goes beyond the mere representation that there is something or other with olfactory properties in one's vicinity, but it is less clear that it is also sufficient. For full-blown perceptual objecthood we also need to recognise the object as persisting through change, which as we shall see, is crucial for object-recognition. As mentioned, the standard notion of perceptual objecthood involves the idea that these entities can-at least to some degree-survive shifts in our own perspective, movements of the objects themselves and certain changes over time. I do not think that figure-ground segregation alone gives us this.

Here are two cases in which it seems that figure-ground segregation applies, but where plausibly there isn't full-blown object perception. First, imagine the experience of standing under a railway bridge, looking up at its underside as the train passes by overhead. ${ }^{4}$ You perceive flashes of light through the gaps between the tracks as the carriages pass by. These flashes of light stand out to us against a background of the environment under the bridge. However, these flashes do not seem to be perceived as perceptual objects, but merely as differences in lighting that jump out in our experience. I do not think we ought to include these flashes as examples of perceptual objects, because if we were to do so, our notion would be extremely weak and wouldn't correspond with any normal usage of the term "object". The notion of objecthood that I am interested in here is one that involves both object individuation and object recognition abilities - the two sets of skills considered to be at the heart of objectperception. Perceiving the flashes doesn't appear to involve object recognition abilities such as tracking, amodal completion or the perception of them as persistent. Thus, nor does it involve perceptual constancies - the flashes aren't perceived as invariant across changes in perspective, but are merely transient figures that capture our attention.

A second example in which there seems to be figure-ground segregation without perceptual objecthood is the experience of an after-image following the perception of a bright light. After-images appear segregated from everything else we experience,

\footnotetext{
4 With thanks to Alistair Isaac (personal communication) for providing this example.
} 
allowing for figure-ground segregation. However, they don't present themselves to us as objects in the world, but instead seem overlaid on our visual field, lacking phenomenal objectivity. They continue to appear to us in whichever direction we look. Thus we do not perceive after-images as invariant amid changing sensory stimulation, because no matter how one's perspective varies, after-images do not provide different sensory input.

Figure-ground segregation is thus not sufficient for perceptual objecthood; there are counter-examples to such a theory. Kubovy and Van Valkenburg's criterion for perceptual objecthood (susceptibility to figure-ground segregation alone) is too weak to capture an important aspect of the perception of objects- the perceived entity's ability to survive changes of perspective and in the object itself. Adding the criterion of the exhibition of constancies will provide a more useful notion of objecthood, which better encapsulates what is generally meant by the term, i.e. something that persists, which we can track, and so forth. Recall O'Callaghan's assertion that “objects perceptibly persist and survive changes" (2016, p. 1273). I shall now discuss the second criterion for perceptual objecthood - the exhibition of perceptual constancies-which will provide us with a more robust notion of perceptual objects.

\subsection{Perceptual constancies}

Contra Kubovy and Van Valkenburg (2001), in my view the exhibition of perceptual constancies is also necessary for the perception of objects. Perceptual constancies are the invariances we perceive amid changing sensory stimulation. For example, I can perceive the invariant size of an object even if my perspective changes, giving rise to an interesting kind of duality to the experience. Consider Peacocke's (1983, p. 12) well-known example of seeing two trees of exactly the same size, one of which is further away than the other. There is a sense in which one of the trees looks bigger in that it takes up more space in the visual field, but there is another clear sense in which the trees are perceived as the same size. The perceiver sees the observer-independent size of the trees. Similarly, Noë (2004, p. 78) observes that although a plate, in some sense, looks elliptical from an angle, the viewer also perceives the objective roundness of the plate. In fact, these observer independent properties are generally much easier to attend to than perspective-dependent appearances; when I look at a plate, it takes some effort to notice the elliptical appearance. A novice artist will struggle to recreate the lines and angles required for an accurate drawing, and may resort to drawing the plate as round. We are able to detect patterns and invariances as our perspective on the world shifts. Perceptual constancies are a requirement for coherent object perception. Part of what it is to perceive an object is to recognise it as the same object from different perspectives. Without such an ability, our perceptions would be extremely confusing - as soon as we moved our eyes, the world around us would appear to change.

Object recognition encompasses a range of skills that are closely connected to these perceptual constancies. It includes capacities to recognise invariance amid change along with semantic categorisation. It involves the ability to recognise tokens of the same type (for example, one might see two tomatoes and recognise a kind of sameness 
among them) and also the ability to perceive objects as the same token object from one moment to the next. Thus, skills such as tracking etc. are taken as object recognition abilities. This aspect of object-perception has been emphasised by a number of olfaction researchers (e.g. Wilson and Stevenson 2003, 2006, 2007; Batty 2010a). Batty's abstract view takes there to be something akin to object recognition in the case of olfaction, even though there is no object individuation.

Perceptual constancies underpin our object-recogition abilities such as tracking, perceiving an object as persistent, and amodal completion. They are needed to perceive an object as maintaining its identity moment to moment, or to categorise objects as belonging to a particular type. Consider an example of tracking an object: I watch a car driving down the street. As I do so, the shape of the car projected onto my retina continually changes as it moves past and different aspects of the car come into view. Despite all of these dramatic perspectival changes, I do not perceive the car itself as changing shape or the colour of the car as changing when the sun reflects off different parts of it. I perceive the car as an invariant three-dimensional object of a particular colour moving in a particular direction. Without these constancy effects, it is not clear that we could track the object amid the flux of changing appearances. Likewise, it seems that these constancies are crucial in perceiving the object as persisting over time. I perceive the car as remaining the same object over time, even as my eyes move and I walk around, and as I see different aspects of the object.

Amodal completion is also closely connected to perceptual constancy. In vision, amodal completion is the perception of an object as complete even though it is not entirely visible. As in the previous cases, this seems to involve an ability to go beyond the immediate sensory evidence to perceive the overall shape of an object. We are presented with a world of whole, invariant objects rather than simply the facing sides of objects with which we are in direct sensory contact. Noë (2006) notes that when one looks at a tomato, although one is only in direct sensory contact with its facing side, "[t]he visual experience of the tomato, when one takes it at face value, presents itself to one precisely as a visual experience as of a whole tomato" (p. 413). This is a case of amodal completion, and one that, according to Noë, depends upon our expectancies regarding how different aspects of the tomato would come into view if we were to change our perspective; our grasp of constancies enables the perception of whole objects despite the limitations of our perspectives. Burge (2010 p. 417) notes that in both tracking cases and amodal completion cases we "perceptually anticipate" that which is not strictly speaking present to our senses. This also suggests a commonality with the constancy cases discussed above. Just as we are able to perceive the round shape of the plate even though this involves a kind of extrapolation from the immediate sensory evidence, we are able to perceive the tomato as whole even though we cannot strictly speaking see all of it at once. Thus, amodal completion is plausibly a special case of our ability to perceive constant aspects of the world - the object is perceived as having an invariant, complete shape despite our sensory limitations. We shall see in Sect. 3.3, that something similar applies in the case of olfaction.

While perceptual constancies are closely connected to object-recognition, there are some aspects of object-recognition that I am hesitant to include as necessary for object perception. For example, object recognition is often also taken to involve implicit object memories - the ability to recognise (at least implicitly) that an object is 
of the same type as a previously experienced object. While object memories are able to influence the way we categorise objects and seemingly can even influence figureground assignments (e.g. Peterson and Gibson 1994), raising interesting questions about how object-recognition and object individuation are interlinked, this aspect of object recognition does not itself appear to be necessary for the perception of objects. After all, if this were the case, it would not be possible to perceive novel objects of which no prior memories are stored. Thus, it is the perceptual constancies underpinning object recognition abilities that are necessary for the perception of objects, rather than (all aspects of) object-recognition itself. Perceptual constancies are required for achieving the initial perception of a coherent object along with feats of recognition such as tracking, persistence, and amodal completion.

Perceptual constancies have been observed across other modalities, and are intimately tied to the notion of perceptual objects across the senses. Researchers have, for example, found various constancies exhibited in auditory perception. It has been observed that the perception of sound streams involves the phenomenon of timbre constancy. Bregman (1990) points out:

A friend's voice has the same perceived timbre in a quiet room as at a cocktail party. Yet at the party, the set of frequency components arising from that voice is mixed at the listener's ear with frequency components from other sources. The total spectrum of energy that reaches the ear may be significantly different in different environments. (p. 2)

We recognise invariance of timbre even though there is also a sense in we hear something quite different in a cocktail party and in a quiet room. Another example is highlighted by Matthen (2010), who, drawing on the work of neuroscientist and musician Levitin (2006), notes that melodies retain their identity even if they are played in a different key. We recognise the sameness of the melodies amid changes. Thus, Matthen takes them to be analogous to the three-dimensional objects we perceive through vision, and describes them as a type of auditory object.

Such perceptual constancies are a necessary condition for object-perception. As noted, without such constancies, we would not be able to recognise an object as persisting amid change. We would merely experience a barrage of changing sensations rather than coherent objects. Thus, if it turns out that olfactory experience does not exhibit such constancies, smell cannot involve the perception of objects in a robust phenomenological sense. However, perceptual constancies alone are not sufficient for the perception of objects as there are types of perceptual constancies that apply to properties, which may not be instantiated by a particular perceived object. In particular, colour constancy may occur in cases where we do not perceive an object. One such case is provided by Kennedy (2007), who suggests that where someone is engulfed in a Ganzfeld fog, although their experience presents to them a uniform, undifferentiated colour, there may still be cases of colour constancy. "The blue as it is presented to me in a Ganzfeld fog could seem a bit thicker or smoother while still appearing to be the same color. In this sort of case, the color character of my experience changes, but I still seem to be aware of the same color. One's experience in a color-constancy Ganzfeldcase would have a significant phenomenological similarity to one's experience of an 
object's color as constant" (p. 315). The experience of the fog doesn't present itself as of an object, and doesn't exhibit figure ground segregation, but it can still involve perceptual constancies.

If a perception involves both boundaries (figure-ground segregation) and constancies, this will be a case of full-blown object-perception in a phenomenological sense. The joint exhibition of these features in perception ensures that the putative object is perceived as discrete, bounded and that it is able to survive changes in one's perspective and movements of the object itself. This is a much stronger notion of objecthood than the non-phenomenological notion offered by Lycan and the weak existentially quantified variety offered by Batty, allowing for the perception of particular objects rather than merely some-or-other undifferentiated object. Using these conditions enables a robust, non-visuocentric notion of perceptual objecthood, which avoids the counter examples faced by Kubovy and Van Valkenburg's (2001) theory. Thus, plausibly susceptibility to figure-ground segregation and perceptual constancies are jointly sufficient for the perception of particular objects, as well as individually necessary. If I am right, then demonstrating that these features of perception are exhibited in olfaction would show there to be olfactory objects in a robust sense.

\section{Does olfaction fulfil the criteria for perceptual objecthood?}

Here I will argue that olfactory experiences often exhibit both figure-ground segregation and perceptual constancies - and thus, we perceive discrete objects through olfaction. As we have seen, figure-ground segregation and perceptual constancies are each necessary for this kind of perceptual objecthood, and thus, if either is missing from olfactory experience, this would show that we do not perceive olfactory objects in a robust phenomenological sense. If there are perceptual constancies but not figureground segregation, this would allow for Batty's weak abstract variety of object, but not allow the perception of particular objects.

I will begin by looking at whether there is a figure-ground structure to olfactory experience. I will suggest that there can be a type of non-spatial figure-ground segregation in olfaction (Sect. 3.1), based on the Gestalt grouping principles, which have been used to provide evidence for perceptual grouping and segregation in vision and audition. Secondly, I will argue that there is also a form of spatial figure-ground segregation that applies in the case of smell (Sect. 3.2). Then in Sect. 3.3, I will focus on perceptual constancies, which are also required for full-blown objecthood. I shall discuss empirical research and olfactory phenomenology, which suggest that there are least two types of perceptual constancy exhibited in olfactory experience, and thus, that olfaction does involve perceptual objects.

\subsection{Figure-ground segregation in olfaction: an aspatial notion based on Gestalt principles}

While there has been research into audition suggesting that figure-ground segregation can occur in a temporal, rather than spatial, manner (Bregman 1990), the phenomenon is often still described in explicitly spatial terms. For example, Batty (2014b) asserts: 
"In those types of experience in which we think of figure-ground segregation as achieved_-vision, audition and touch, for example — we do so on the basis of the richness of its spatial representation" (p. 10). Visual experience provides a paradigmatic example of this kind of rich spatiality, presenting us with clearly bounded, spatially arrayed objects that we distinguish from each other and from the background. Olfaction doesn't ostensibly do this and as mentioned above, many researchers consider olfaction to be aspatial or spatially undifferentiated (Lycan 2000; Matthen 2005; Wilson and Stevenson 2006; Batty 2011, 2014b). It therefore remains highly controversial whether olfaction achieves figure-ground segregation.

Researchers that have argued in favour of olfactory figure-ground segregation include Wilson and Stevenson (2006, 2007), Carvalho (2014) and Young (2016). Wilson and Stevenson $(2006,2007)$ and Carvalho (2014) have broadly similar accounts of olfactory figure-ground segregation. They suggest that we should understand figureground segregation in smell as being an ability to perceive an individual odour against a background of other odorants. Carvalho says: "for one of these odors to be experienced as such, as the odor that it is, the olfactory system needs to be able to extract a very complex blend against a background of irrelevant odorants and competing olfactory objects" (2014, p. 63). For example, intuitively, when I walk into a coffee shop, I can perceive a unified coffee odour despite there also being a complex blend of odorants from cleaning products, food, perfume and so forth. We perceive the coffee as a "unitary percept" (Wilson and Stevenson 2006), which then stands out against the other odorants in the air. Batty takes these sorts of arguments to rely on the idea that the coffee odour has "experiential prominence" (2014a, 2014b), and argues that her own approach is equally well-placed to explain the experiential prominence of the coffee smell.

Recall, Batty believes that olfactory experience is just too "smudgy" to involve bounded, discrete objects. Rather than particular objects, olfactory experience only represents olfactory properties instantiated by some-or-other object "here" in one's vicinity. She takes "here" to be an undifferentiated location, claiming that we do not distinguish where the smell is instantiated from where it is not (2010a, p. 9). The content of olfactory experience is thus indexical (in that odours are experienced as "here") and abstract in the sense that rather than representing particular objects they simply represent "that there is something or other here with certain properties" (Batty 2011, p. 170). She takes olfactory experience itself to be silent on which odour instantiates which olfactory property (since she denies there is object individuation in olfaction). If one can smell coffee and perfume, for Batty the experience will merely report that it smells perfumey and coffee-like here (see 2010a, p. 534; 2011, pp. 166-167). If certain aspects of one's experience are especially prominent (say the coffee smell sticks out to the perceiver over the perfumey smell), for Batty, this will be a case of experiential prominence, which need not be understood as a kind of figure-ground segregation. This could merely be a case of certain properties being experienced as more prominent than other properties of some-or-other object, and this could be explained in terms of attention/expectation, or the mechanisms of learning and memory, without the need to attribute figure-ground effects and discrete objects to the experience (2014a, p. 237). Thus, Batty claims that her abstract view is, prima facie, equally well equipped to explain our olfactory experiences. 
If Batty is right, how ought we decide whether olfactory perception involves figureground segregation? It appears that relying upon experiential prominence, as Wilson and Stevenson $(2006,2007)$ and Carvalho (2014) do, will alone not suffice to establish this. As mentioned, much of the motivation for object-talk in perception is the interesting set of commonalities among our perceptual processes and the entities we perceive as grouped. The Gestalt psychologists uncovered a range of principles that govern when we perceive stimulus features as bounded and discrete (and thus exhibiting figure-ground segregation). These principles may be useful in determining whether figure-ground segregation is exhibited in the olfactory case because if they apply, this would suggest that groupings and segregations analogous to those in the other senses are occurring. A robust notion of olfactory figure-ground segregation ought to bear a resemblance to the way separations of figure and ground occur in the other senses, especially as people's intuitions on this point seem to differ a great deal.

The Gestalt psychologists placed phenomenal experience at the heart of their approach to the mind. They believed that we do not first experience disjointed sensations, but rather an organised field of structured wholes, segregated from everything else around them, and sought to uncover stimulus features that determine how the perceptual field is organised. Wertheimer (1923/1938) outlined aspects of a stimulus that influence perceptual organisation, giving rise to a range of grouping principles such as similarity, proximity, common fate and good form (the law of Prägnanz). For example, in the case of the principle of similarity: items that are similar in colour, shape, texture, etc. are more likely to be visually grouped. The Gestalt psychologists found that these grouping principles exerted a significant influence on the way that people perceive visual displays (e.g. Wertheimer 1923/1938), and it has been found that all of these principles are also applicable to auditory perception (e.g. Bregman 1990).

If olfaction only allowed the perception of nebulous properties (Matthen 2005) or merely the weak sense of existentially quantified objecthood advocated by Batty, olfactory experience would not involve structured wholes segregated from everything else around them. If the Gestalt principles of perceptual organisation apply to the objects/features we perceive through smell, this would be a good reason for describing olfaction as exhibiting figure-ground segregation. It would demonstrate clear similarities to the pre-established notions of visual and auditory figure-ground segregation, and would suggest that perceptual organisation in olfaction occurs in ways analogous to the other senses. In fact, there is evidence that, even disregarding any potential spatial import in olfactory experience, several of these principles apply. ${ }^{5}$

\footnotetext{
5 Other Gestalt principles may well also apply in the olfactory case, but there is limited research in this area. The principle of common fate says that we are more likely to perceive elements that move together as being grouped. Bregman (1990, p. 249), however, suggested that this principle can be expanded to include elements that change together in proportional and synchronous ways. He says that would show this principle to be applicable to audition, in cases where, for example, two frequency components changed synchronously by proportional amounts and were therefore likely to be perceived as grouped. Olfactory experiences, like auditory ones, rely heavily on the temporal extension, unfolding over time in important ways. It would be interesting to see if something analogous occurs in the case of smell. It is plausible that experiencing odorants as changing in a synchronous manner would result in a greater likelihood of grouping, but as far as I am aware there is no research exploring this issue. The applicability of at least some of the principles does, nevertheless, add empirical support to the view that olfaction involves figure-ground segregation.
} 
Principle of Similarity The principle of similarity states that we tend to group similar aspects of a stimulus together. There are some issues in assessing how we are to determine whether items are similar, but generally it is assumed that parts of a stimulus that, for example, share the same colour or shape are more similar (at least in these respects) than those that don't. For example, in the case of vision, two red dots are more likely to be grouped into a structured whole than a red and a blue dot. These sorts of specific cases of similarity are taken to be instantiations of a broader principle of similarity. ${ }^{6}$ There is evidence that something analogous is applicable in the case of olfaction. Research shows that mixtures of similarly smelling components are more likely to be perceived as having unified, novel olfactory qualities than mixtures of dissimilar components. In cases where the components smell dissimilar it is generally easier to individuate them and perceive them as separate than in the cases where the individual odorants smell similar (Wiltrout et al. 2003; see Young 2016 for further discussion). This seems to be an instance of perceptual grouping based on similarity, and lends weight to the idea that figure-ground segregation occurs in olfaction.

Principle of Proximity Another of the Gestalt Principles is the law of proximity, which states that elements that are closer together are more likely to be perceived as grouped. Generally, in the visual case, this is simply measured by manipulating distances between aspects of the input image. For example, studies show that dots that are clustered closely together are more likely to be perceived as grouped together into a unified figure (e.g. Compton and Logan 1993). In audition, Bregman (1990) suggests that an analogue of this is separation in time (p. 19). One manifestation of this principle in audition is that concurrent onset of sounds provides a good indication that they are members of the same stream, while different start times can signal different streams (O'Callaghan 2008, p. 822). Separation in time seems to also be relevant in the olfactory case, as there is evidence that suggests that (as in audition) concurrent onset of olfactory stimuli increases the chance of us perceiving them as belonging together. Studies show that in multimodal flavour-smell interactions, simultaneous onset makes them more likely to be integrated (Pfeiffer et al. 2005; Stevenson 2014). While this hasn't been directly tested in the non-multisensory olfactory case, it is very plausible that something similar would apply. I take this to be indirect evidence of the law of proximity applying in the case of olfaction.

Principle of Good Form Another principle that is worth considering is the principle of good form or "Prägnanz"-an overarching Gestalt principle that is often said to

\footnotetext{
6 These kinds of simple visual examples have the potential to be misleading. In the case of grouping together dots, the individual dots may themselves be perceived as figures against a background in some cases (presumably based in part on further grouping principles), but they are also often perceptually combined into larger perceived objects. Thus, it may be ambiguous whether we perceive an individual dot as a figure against a background or the larger grouping of dots as a figure (perhaps like cases of bi-stable figure-ground perception such as Rubin's "vase-or-face" image, which can be perceived according to two distinct figureground interpretations). In many cases, however, there isn't such an ambiguity. Plausibly, part of the reason we perceive a tomato as a unified, bounded object is because of the similar shades of red across different parts of it. In such cases we don't perceive one patch of the tomato as itself a figure against a background, even though it may be possible to attend to one patch of it. Likewise, in the case of olfactory grouping by similarity, the perception is of a unified odour, and even if it may be possible in some instances to attend to different aspects of the odour, these aspects don't seem to be segregated from the whole. Thanks to an anonymous referee for pushing me on this issue.
} 
encompass the other grouping principles. This states that we are more likely perceptually group together assemblies of parts that are coherent, balanced, simple, and so forth. We generally group together elements into the simplest and most internally coherent figures that are compatible with the available sensory information. Intuitively, good form is something that can be achieved in the olfactory domain. Perfumery provides an example of this, and suggests that good form aids in the grouping of odours. Perfume manufacturers seek to develop well-balanced fragrances, combining top, middle and base notes to obtain a perfume accord. An accord is a blend of notes that produces a harmonious odour, in which the notes lose their individual identity and are perceived as unified. Perfume accords are plausibly bounded and segregated from other scents, and good form seems to be an important factor in achieving this. The balance, harmony and internal coherence exhibited by these accords is crucial to the perception a unified scent.

These examples highlight that several of the Gestalt principles apply in the case of olfaction, influencing whether we perceive olfactory features as unified and discrete. This doesn't look like mere experiential prominence but rather, olfactory experience seems to be organised in ways analogous to in the other senses. Thus, there is good reason to apply the notion of figure-ground segregation to olfactory perception. Importantly, if the evidence I have provided does indicate that the several of the Gestalt principles apply to smell (thus providing evidence for figure-ground segregation), they seem to apply in a non-spatial manner. In the next section, I will argue that in addition to this type of figure-ground segregation, olfaction also exhibits a form of spatial figure-ground segregation.

\subsection{Figure-ground segregation and the spatiality of olfaction}

We have seen that there are reasons to think that, as in the auditory case, there is a nonspatial form of figure-ground segregation in olfaction. However, some researchers have rejected the idea of non-spatial figure-ground segregation, suggesting that the notion should be reserved for instances of spatial discrimination (which is usually thought to preclude olfaction). Batty (2014b) argues that we need a spatial account of figure-ground segregation in order to make interesting comparisons between human and non-human olfactory perceptions. In particular, it is clear that some animals have a directional sense of smell. Batty notes that the hammerhead shark's sense of smell enables it to immediately turn in the direction of the source of a blood odour (p. 10). Moreover, its nasal cavities are far apart, giving it a stereo sense of smell. According to Batty, the shark's sense of smell can rightly be said to involve a kind of (spatial) figure-ground segregation, while ours cannot. She says, "If we are to account for the difference between us and the hammerhead, then, we require the spatial notion of figure-ground segregation" (ibid.). This ability to immediately detect the direction from which the blood arrives is, she claims, analogous to our ability to detect the location of a sound in audition (ibid.).

Batty's argument is primarily pragmatic; it is based on the alleged utility of reserving the term "figure-ground segregation" for spatial types of perception. Extending the term to non-spatial cases, she thinks, threatens its usefulness in accounting for 
differences between varied types of perception (such as human olfaction and that of the shark). As we have seen, Bregman (1990) and others have claimed that there are important notions of figure-ground segregation that aren't reliant on spatial features, allowing us, for example, to make sense of how we perceive distinct sound streams. While audition does, as Batty notes, enable us to spatially locate objects and events in the environment, it also appears to be governed by a myriad of Gestalt grouping principles that are heavily reliant on temporal and non-spatial features. These principles provide evidence of non-spatial figure-ground effects in audition. Furthermore, we have seen that at least some of these Gestalt principles apply to olfaction also. Nevertheless, even if one were to reserve the notion of figure-ground segregation for spatial cases, we will see that it can still apply to olfactory experience. Thus, here I also disagree with Wilson and Stevenson (2006, 2007) and Carvalho (2014), who think only non-spatial figure-ground segregation occurs in olfaction, and others such as Lycan (2000) and Matthen (2005) who reject any kind of figure-ground segregation in olfaction. In what follows, I will respond to Batty and argue that spatial olfactory figure-ground segregation is at least sometimes achieved. In order to see that olfaction does provide us with spatial information, we need to first understand that bodily activity is required to have any kind of olfactory experience and that tactile inputs can give rise to olfactory phenomenology.

A clear sense in which olfaction involves (a minimal kind of) bodily movement is through the act of sniffing. Empirical research demonstrates that the act of inhalation is necessary to have any olfactory experience whatsoever. Proetz (1941) highlighted the necessity of airflow through the nostrils by pouring a solution of eau de cologne into the nostrils of his students, noting that this did not give rise to an olfactory perception: "Although it obviously reaches his olfactory area he will not detect the odor" (p. 366). Further evidence for the necessity of the inhalation comes from Bocca et al. (1965), who intravenously injected odorants into subjects. Odorants were delivered to the epithelium via the blood stream, but subjects did not perceive any odour except for when they sniffed and breathed normally through the nose. Thus, the act of inhalation is crucial for olfactory experience, with some taking the sniff to be "as integral to olfactory perception as the eye movement is to visual perception" (Mainland and Sobel 2006, p. 1). In addition, evidence suggests that multiple sniffs are needed to differentiate odours (Mainland and Sobel 2006). Thus, we ought to acknowledge the role of this kind of temporally extended olfactory exploration in considering whether figure-ground segregation is achieved.

Active sniffing also appears to offer us more spatial information than has generally been supposed. A conservative endorsement of spatiality in olfactory experience comes from Louise Richardson (2013). She argues that the act of sniffing causes us to perceive smell in an exteroceptive manner: we perceive odours as being brought into the nose from the outside. In this way, smell differs from bodily sensations like pain, for example. Richardson doesn't think that distance and direction are represented in olfactory experiences, claiming that they are more akin to the tactile experience of a breeze blowing against your face: "One is not aware of some distance that the air that touches your face has travelled. But nevertheless, it does seem as if the breeze is coming to the body from somewhere beyond it—from without" (p. 411). This is an interesting analogy because it appears to actually highlight nearly the opposite of 
what Richardson claims. We can learn about direction (although perhaps not distance) from air blowing into your face. Wind might hit my left cheek, but not my right cheek, giving me information about which direction it is coming from. Something similar might hold in certain cases of olfaction.

In fact, Roberts (2015) uses this line of thought—and a similar example —in arguing in favour of spatial content in olfaction. He asserts:

Firstly, consider that some odours arrive on the breeze, and are experienced as such. Directional ventilation can add a dimension of spatial content that is missing in olfactory encounters with static air, permitting the subject to smell where an odour is coming from. A scent that is carried by a current that is felt upon the left hand side of one's face can, plausibly, be perceptually localised to that direction... (p. 408)

The example of odours carried on a breeze seems to show that there can be directional content in olfaction. This goes beyond the exteroceptivity that Richardson highlights, illustrating the beginnings of a more robust account of spatial olfaction.

As well as the case of odours arriving on a breeze, there are empirical studies that indicate that we can gain some directional information through smelling by stimulation of the trigeminal nerve (Kobal et al. 1989) - a nerve involved in activity such as chewing and responsible for (what are usually considered to be) tactile and pain sensations in the face. It might be protested that these directional experiences aren't truly olfactory, given the involvement of trigeminal or other types of tactile (in the breeze case) stimulation. However, as Roberts (ibid.) points out, it seems to be a mistake to think that there is ever "pure olfaction" devoid of input from the other senses (p. 418, fn. 17). As we have seen, the act of smelling always involves bodily movement in the form of the sniff, which like the experience of a breeze hitting us, involves a degree of tactile stimulation. If we agree with Richardson that our olfactory experiences are exteroceptive, this seems to crucially involve the experience of the inhalation of air through the nostrils. This exteroceptivity is a vital aspect of olfactory phenomenology, but one that depends upon tactile input.

Likewise, many smells rely on trigeminal stimulation for their perceived quality. For example, the cool scent of menthol, the pungency of ammonia and the acridity of burning rubber are all reliant on trigeminal stimulation. Yet these do seem to be features of odours, we detect them by sniffing, and they seem to present themselves as olfactory properties. Our experiences of these properties seem to undermine the idea that we can neatly carve off olfactory phenomenology from tactile phenomenology. It might be thought that considering anosmia, where patients have lost their sense of smell, would allow us to isolate the trigeminal from the olfactory aspects of experience. However, in fact this doesn't provide a clear way to separate the two because anosmia also comes with a decrease in trigeminal sensitivity (Hummel et al. 1996), illustrating the tight connection between olfactory and trigeminal stimulation in smell. Trigeminal stimulation is ubiquitous in our normal olfactory experiences and the vast majority of odorants also stimulate the trigeminal nerve (e.g. Wysocki et al. 2003).

There have also been interesting experiments demonstrating that humans have a capacity for tracking odour trails. Porter et al. (2007) show that we are in fact able 
to track odour trails using smell alone (participants were blindfolded, given soundblocking earmuffs etc.). ${ }^{7}$ Accuracy dropped significantly when the subjects had one nostril taped up, and when a device was used to combine airflow so both nostrils received the same information. This suggests that our bilateral nostrils play a role in the spatial information derived from olfaction: "Here we find that mammals performing a scent-tracking task, freely able to move their nose and sample the olfactory environment in real time, reap added benefit from sampling via their two spatially offset nostrils" (p. 29). They conclude that we are able to spatially localise odorants. In this case, it is very plausible that the subjects experience the scent trail as a figure against a background - a case of olfactory figure-ground segregation that is achieved spatially. Whether we move our bodies to seek out the scents that we inhale, or whether the air is brought to us on a breeze, we can garner spatial information from olfaction.

Now we are in a position to re-consider Batty's (2014b) shark example. Batty infers a spatial type of perception from the shark's abilities, taking hammerhead sharks to have a directional sense of smell because they have the ability to immediately turn in the direction of the source of a blood odour, for example (p. 10), as well as noting that their offset nostrils allow stereo smell. Yet Batty's 'abstract' account of human olfaction requires that we assess the phenomenology of a motionless perceiver. She says: "If we bracket information gained from movement and any other sensory modality, and consider olfactory experience at-a-time, then we see that any locatedness of these properties—other than simply 'here'-goes as well'" (2010a, p. 9). As we have seen, the idea that olfactory properties are simply instantiated 'here' is central to her account, highlighting that she believes that bracketing off movement and information from the other senses is the correct approach to understanding human olfaction. Batty says "it would be strange to conclude that the hammerhead's olfactory experiences are to be evaluated according to one notion of figure-ground segregation, while ours are not" (2014b, p. 10). However, human scent tracking also involves differential stimulation of the nostrils and Porter, et al.'s research seems to suggest clear similarities in the perceptual abilities of humans and sharks. Participants in the study moved their heads in such a way as to follow the odour trail, from which we can infer the sort of spatial phenomenology that Batty claims is the preserve of non-human animals. Thus, there doesn't seem to be a reason to accept evidence for figure-ground segregation from a shark's abilities but not from those of a human. If the hammerhead shark's sense of smell is said to involve figure-ground segregation, it seems that (at least) in certain circumstances human olfaction does so too.

In fact, a key role of olfaction is to spatially localise the sources of odours, and exploratory sniffing also allows us to track the direction from which some odours

\footnotetext{
7 Again, some researchers are likely to argue that this isn't an example of "pure" olfaction. Lycan, who, as I have mentioned, claims olfaction is aspatial, would disagree with this account of spatial content in olfaction because he thinks that spatial information derived from sniffing while moving our heads or bodies wouldn't count as spatial content from olfaction itself (personal communication). His focus is only on whether there is spatial content in smell when we don't move around. However, once we recognise that some bodily movement is a requirement for having any olfactory experience whatsoever, it is a small step to accept that ordinary olfactory experience involves exploratory sniffing. We move our heads towards roses to smell them, we move away from bad smells, we lift the coffee cup towards our noses to smell its odour, and so forth.
} 
arrive. We don't only perceive odours as exteroceptive through olfaction, but can also learn about direction and other spatial features of odours, which may assist us in segregating them. Thus, contra researchers like Batty (2011, 2014b), Lycan (2000) and Matthen (2005) it appears that we do have spatial discriminatory abilities in olfaction. By adopting this approach to olfaction, we can also take insights from the processbased accounts of olfaction (mentioned in Sect. 1) while retaining a robust notion of objecthood. Barwich (2014) says that we ought to reject the notion of perceptual objects because researchers treat them as static units of analysis, independent of perceptual processes (p. 264). However, with the proposed approach we can see that perceptual objects are crucially dependent upon perceptual processes. Without bodily movement, we cannot perceive objects at all, and moving our heads and bodies can give us rich spatial information about odours, allowing us to spatially segregate them from complex mixtures of odorants in the air.

The preceding discussion's emphasis on the role of tactile and trigeminal stimulation in olfactory phenomenology opens up interesting questions about whether we ought to take olfactory experience to be multimodal (perhaps similar to how flavour perception is often understood). In my view, there are three main ways to construe olfaction here: (a) as a multimodal type of perception involving olfactory, tactile and trigeminal sense modalities, (b) as a unimodal type of perception, which takes the tactile/trigeminal involvement to merely influence olfaction rather than forming part of the olfactory experience itself, or (c) as a unimodal type of perception that takes the tactile and trigeminal involvement to form a constitutive part of a unified olfactory sense modality/perceptual modality. How this question is answered will depend on the way in which the senses are individuated. ${ }^{8}$ There have been extremely wide-ranging approaches to this issue. Traditionally, most approaches have based individuation on either the phenomenal character of a sense experience, types of receptors, the sense organ involved or the representational content of the experience (Macpherson 2011b), and others have suggested we individuate the senses on the basis of types of perceptual activity (Matthen 2015) or societal convention (Nudds 2004). These different strategies for individuating the senses will result in different answers to the question of whether olfactory experience is multimodal. ${ }^{9}$ Although these questions goes beyond

\footnotetext{
8 See Macpherson (2011a) for an introduction to different approaches to individuating the senses and see Richardson (2013) for discussion of the exteroceptivity of olfaction and its implications for the debate on individuating the senses.

9 For example, if the senses are individuated on the basis of the phenomenal character of the associated experiences or on the basis of perceptual activity (e.g. sniffing, tracking odour sources etc.), olfactory experiences could be considered unimodal in character (option c). With this approach, the tactile and trigeminal elements would play a constitutive role in the sense of smell (if it is agreed that there is no clear way to abstract olfactory phenomenology/types of worldly engagement away from the tactile/trigeminal aspects). Whereas, if the senses were individuated (for example) on the basis of the type of receptors that are being stimulated, this would most likely lead to either option (a) or (b) depending on exactly how one thinks that senses individuated on the basis of receptor types relate to conscious olfactory experience. If one thinks that olfactory experience depends (constitutively) upon all the receptors that are characteristically stimulated when one smells something, olfactory experience would be multimodal, involving several different sense modalities (option a). If one thinks that olfactory experience is merely influenced causally by tactile and trigeminal stimulation, but that there is not a constitutive relationship between them, then olfactory experience would not be multimodal (option b). For what it's worth, my own preference on this issue is for option (c), treating olfaction as a unified perceptual system individuated on the basis of bodily activity.
} 
the scope of the current discussion, this would be a worthwhile avenue for future exploration.

With the account I have put forward, odours are presented to us as spatiotemporally extended entities. Even though they have less clearly specified spatial boundaries than the objects presented in, say, vision, they do allow spatial differentiation when we actively engage in the activity of smelling. There is good evidence that figure-ground segregation applies in olfaction: it is possible to discriminate odours both on the basis of non-spatial and spatial factors. Batty (2014b) argues against the presentation of particular objects in olfaction because of the alleged lack of figure-ground segregation, so a key argument in favour of the abstract view fails. Similar considerations would hold against the feature-based view prevalent in the olfactory sciences; we have seen that there is good reason to think that olfaction involves the experience of discrete structured wholes, rather than disjointed properties. However, to assess whether there are olfactory objects, we must also determine whether olfaction involves perceptual constancies.

\subsection{Perceptual constancies in olfaction}

In the previous sections, I discussed two ways in which figure-ground segregation is exhibited in olfaction. We saw that figure-ground segregation alone, however, is not sufficient for the exhibition of particular objects in experience. If perceptual constancies are also exhibited in olfactory experiences, olfaction will meet the criteria for the perception of particular objects. For robust object perception, I have claimed, we require more than a mere moment-by-moment individuation of odours; we also need these odours to retain their identity across change (involving object recognition capacities).

It has been claimed that olfaction doesn't involve perceptual constancies (e.g. Burge 2009). However, there is evidence to the contrary. One type of constancy in olfaction is our ability to "fill in" compounds. We are able to perceive odours as the odour-type that they are, even if they are corrupted or missing elements due to poor perceptual conditions (Barnes et al. 2008; see Carvalho 2014 for a discussion of this type of constancy). We perceive a coffee odour as a coherent whole, which retains its identity, even if the precise odorants that stimulate the nasal cavity don't include all 600 chemical elements that typify a coffee odour. We recognise it as a coffee odour even if the particular compounds that we are in contact with vary. It is thought that this is a result of object-recognition processes (Stevenson 2011, p. 1892). This seems analogous to the sort of visual object-recognition that allows us to see objects as complete, even though we only have direct sensory contact with their facing sides and in cases where they are partially occluded.

Another type of perceptual constancy in olfaction relates to intensity. The size of sniff that one takes has a significant effect on the neural response of the olfactory nerve, but does not result in significant changes to the apparent intensity of an odour (Teghtsoonian and Teghtsoonian 1982). We seem able to perceive an invariant feature of an odour-its intensity-even though our perspectives on the world change. This appears to be related to an awareness of how one's own sniffing activity will influence 
the olfactory stimuli that reaches the olfactory epithelium. Crucially, when the changes to airflow rate are not controlled by the subject, there is no such constancy effect and there is a perceived change to odour intensity, highlighting again the importance of bodily activity (here, in terms of the effect of effort expended when sniffing) in olfactory perception (Hahn et al. 1994). There are perceptual constancies in olfaction as in the other sensory modalities.

In Sect. 2.2 I noted that in the cases of perceptual constancy exhibited in visual experience, there is generally a kind of duality to the phenomenology of the experience. There is a sense in which one tree looks smaller than the other, and the plate looks elliptical, but in another more robust sense, the trees look the same size and the plate looks round. Could something like this apply to the olfactory constancies discussed above? In the case of intensity constancy, it is clear to me that it does. There is a sense in which if I take a big sniff of coffee and a small sniff of coffee, I can notice that there is, in a way, a different intensity to the experience, but at the same time I perceive the coffee odour as having an invariant intensity.

In the case of "filling-in", it is a little more difficult to see how this kind of duality could apply, but I still think that there is a case to me made for something like this applying to olfactory phenomenology. This case is analogous to the visual case of seeing an object as complete even though we only see its facing side, or it is partially occluded by something. In the visual case, there is a sense in which the object appears as a whole object even though in another sense we recognise that we only see part of it. Perhaps in the case of olfaction, one could recognise that not all of the properties typical of the coffee odour are apparent at once (picking out, say, the fruitiness and yet needing to move closer to coffee cup to pick out the chocolate notes), and yet still also recognise the coffee odour as complete and unified in another sense. Plausibly, an expert coffee taster would be better at picking out these perspective-dependent olfactory properties, just as an experienced artist is better able to pick out the angles and shapes apparent from a perspective. Yet, the unified coffee odour-like the objective shape of the plate-will generally be easier to pick out than the particular properties apparent at each moment.

If we accept the role of tactile stimulation and bodily movement in olfactory phenomenology, we can see that olfaction involves both figure-ground segregation and perceptual constancies, and thus fulfils the criteria I laid out for a robust type of perceptual objecthood. This object-based approach improves upon the abstract and feature-based approaches, which usually rely on the claim that olfaction is aspatial and can't exhibit figure-ground effects. With my suggested approach, we can appreciate the important ways in which olfaction is similar to other varieties of perception, providing us rich information about the world.

\section{Conclusion}

In this paper I argued that olfaction allows the perception of particular, discrete objects in the form of odours. I argued that figure-ground segregation and perceptual constancies are the hallmarks of the perception of particular objects, providing individually necessary and jointly sufficient conditions. We saw that there is empirical evidence 
that the Gestalt principles of similarity, proximity and good form apply in the case of olfaction, suggesting that there is a non-spatial variety of figure-ground segregation exhibited by smell. Additionally, however, we saw that olfaction involves a second, spatial type of figure-ground segregation and olfaction is experienced in spatial manner. The figure-ground structure and spatiality of olfactory experience is especially evident when we pay attention to the role of tactile stimulation and bodily movement in olfactory experience. Furthermore, there is empirical and phenomenological evidence of perceptual constancies in olfaction, which together with figure-ground segregation, fulfils the criteria for a robust kind of perceptual objecthood that goes beyond the weak, abstract notion of objecthood or the perception of nebulous features.

Acknowledgements I would like to thank Dave Ward, Alistair Isaac, Jonny Lee, Franklin Jacoby, Henry Schiller and Joe Dewhurst for many helpful comments and fruitful conversations. I am also grateful to two anonymous reviewers for their extremely constructive suggestions.

Open Access This article is distributed under the terms of the Creative Commons Attribution 4.0 International License (http://creativecommons.org/licenses/by/4.0/), which permits unrestricted use, distribution, and reproduction in any medium, provided you give appropriate credit to the original author(s) and the source, provide a link to the Creative Commons license, and indicate if changes were made.

\section{References}

Barnes, D., Hofacer, R., Zaman, A., Rennnaker, R., \& Wilson, D. (2008). Olfactory perceptual stability and discrimination. Nature Neuroscience, 11, 1378-1380.

Barwich, A.-S. (2014). A sense so rare: Measuring olfactory experiences and making a case for a process perspective on sensory perception. Biological Theory, 9(3), 258-268.

Batty, C. (2010a). A representational account of olfactory experience. Canadian Journal of Philosophy, 40, 511-538.

Batty, C. (2010b). What the nose doesn't know: Non-veridicality and olfactory experience. Journal of Consciousness Studies, 17, 10-17.

Batty, C. (2011). Smelling lessons. Philosophical Studies, 153(1), 161-174.

Batty, C. (2014a). Olfactory objects. In D. Stokes, M. Matthen, \& S. Biggs (Eds.), Perception and its modalities (pp. 222-246). New York: Oxford Scholarship Online. Retrieved February 15, 2016, from http://www.oxfordscholarship.com.ezproxy.is.ed.ac.uk/view/10.1093/acprof:oso/ 9780199832798.001.0001/acprof-9780199832798-chapter-10.

Batty, C. (2014b). The illusion confusion. Frontiers in Psychology, 1-11. Retrieved January 29, 2016, from https://doi.org/10.3389/fpsyg.2014.00231.

Bocca, E., Antonelli, A., \& Mosciaro, O. (1965). Mechanical co-factors in olfactory stimulation. Acta Oto-Laryngologica, 59, 243-247.

Bregman, A. S. (1990). Auditory scene analysis: The perceptual organization of sound. Cambridge, MA: MIT Press.

Bregman, A. S., \& Campbell, J. (1971). Primary auditory stream segregation and perception of order in rapid sequences of tones. Journal of Experimental Psychology, 89, 244-249.

Burge, T. (2009). Perceptual objectivity. Philosophical Review, 118(3), 285-324.

Burge, T. (2010). Origins of objectivity. New York: Oxford University Press.

Carvalho, F. (2014). Olfactory objects. Disputatio, 6(38), 45-66.

Cavedon-Taylor, D. (forthcoming). Odors, objects and olfaction. American Philosophical Quarterly, 1-17.

Compton, B. J., \& Logan, G. D. (1993). Evaluating a computational model of perceptual grouping by proximity. Perception \& Psychophysics, 53(4), 403-421.

Cooke, E., \& Myin, E. (2011). Is trilled smell possible? How the structure of olfaction determines the phenomenology of smell. Journal of Consciousness Studies, 18(11-12), 59-95.

Griffiths, T. D., \& Warren, J. D. (2004). What is an auditory object? Nature Reviews Neuroscience, 5 , 887-92. 
Hahn, I., Scherer, P. W., \& Mozell, M. M. (1994). A mass transport model of olfaction. Journal of Theoretical Biology, 162(2), 115-128.

Hummel, T., Barz, S., Lotsch, J., Roscher, S., Kettenmann, B., \& Kobal, G. (1996). Loss of olfactory function leads to a decrease of trigeminal sensitivity. Chemical Senses, 21, 75-79.

Kennedy, M. J. (2007). Visual awareness of properties. Philosophy and Phenomenological Research, 75(2), 298-325.

Kobal, G., Van Toller, S., \& Hummel, T. (1989). Is there directional smelling? Experientia, 45, 130-132.

Kubovy, M., \& Van Valkenburg, D. (2001). Auditory and visual objects. Cognition, 80, 97-126.

Levitin, D. J. (2006). This is your brain on music: The science of a human obsession. New York: Dutton.

Lycan, W. G. (1996). Layered perceptual representation. Philosophical Issues, 7, 81-100.

Lycan, W. G. (2000). The slighting of smell. In N. Bhushan \& S. Rosenfeld (Eds.), Of minds and molecules: New philosophical perspectives on chemistry (pp. 273-290). New York: Oxford University Press.

Macpherson, F. (2011a). Introduction: Individuating the senses. In F. Macpherson (Ed.), The senses: Classic and contemporary philosophical perspectives (1st ed., pp. 3-43). New York: Oxford University Press.

Macpherson, F. (2011b). Taxonomizing the senses. Philosophical Studies, 153, 123-142.

Mainland, J., \& Sobel, N. (2006). The sniff is part of the olfactory percept. Chemical Senses, 31, 181-196.

Matthen, M. (2005). Seeing, doing, and knowing. Oxford: Oxford University Press.

Matthen, M. (2010). On the diversity of auditory objects. Review of Philosophy and Psychology, 1(1), 63-89.

Matthen, M. (2015). The Individuation of the Senses. In M. Matthen (Ed.), The Oxford handbook of philosophy of perception. Oxford: Oxford University Press.

Noë, A. (2004). Action in perception. Cambridge, MA: MIT Press.

Noë, A. (2006). Experience without the head. In T. Gendler \& J. Hawthorne (Eds.), Perceptual experience (1st ed., pp. 411-434). New York: Oxford University Press.

Nudds, M. (2004). The significance of the senses. Proceedings of the Aristotelian Society, 104(1), 31-51.

O'Callaghan, C. (2008). Object perception: Vision and audition. Philosophy Compass, 3, 803-829.

O'Callaghan, C. (2016). Objects for multisensory perception. Philosophical Studies, 173(5), 1269-1289.

Peacocke, C. (1983). Sense and content. Oxford: Oxford University Press.

Peterson, M., \& Gibson, B. (1994). Must figure-ground organization precede object recognition? An assumption in peril. Psychological Science, 5, 253-259.

Pfeiffer, J. C., Hollowood, T., Hort, J., \& Taylor, A. J. (2005). Temporal synchrony and integration of sub-threshold taste and smell signals. Chemical Senses, 30(7), 539-545.

Porter, J., Craven, B., Khan, R. M., Chang, S.-J., Kang, I., Judkewitz, B., et al. (2007). Mechanisms of scent-tracking in humans. Nature Neuroscience, 10, 27-29.

Proetz, A. (1941). Applied physiology of the nose. St Louis, MO: Annals Publishing Company.

Richardson, L. (2013). Sniffing and smelling. Philosophical Studies, 162, 401-419.

Roberts, T. (2015). A breath of fresh air: Absence and the structure of olfactory perception. Pacific Philosophical Quarterly, 97(3), 400-420.

Stevenson, R. J. (2011). Olfactory illusions: Where are they? Consciousness and Cognition, 20, 1887-1898.

Stevenson, R. J. (2014). Object concepts in the chemical senses. Cognitive Science, 38(7), 1360-1383.

Teghtsoonian, R., \& Teghtsoonian, M. (1982). Perceived effort in sniffing: The effects of sniff pressure and resistance. Perception \& Psychophysics, 31(4), 324-329.

von der Heydt, R. (2015). Figure-ground organization and the emergence of proto-objects in the visual cortex. Frontiers in Psychology, 6, 1-10.

Wertheimer, M. (1923/1938). Laws of organization in perceptual forms. In W. Ellis (Ed.), A source book of Gestalt psychology (pp. 71-88). London: Routledge \& Kegan Paul.

Wilson, D. A., \& Stevenson, R. J. (2003). The fundamental role of memory in olfactory perception. Trends in Neurosciences, 26(5), 243-247.

Wilson, D. A., \& Stevenson, R. J. (2006). Learning to smell: Olfactory perception from neurobiology to behavior. Baltimore, MD: Johns Hopkins University Press.

Wilson, D. A., \& Stevenson, R. J. (2007). Odour perception: An object-recognition approach. Perception, 36(12), 1821-1833.

Wiltrout, C., Dogra, S., \& Linster, C. (2003). Configurational and nonconfigurational interactions between odorants in binary mixtures. Behavioural Neuroscience, 117(2), 236-45.

Wysocki, C. J., Cowart, B. J., \& Radil, T. (2003). Nasal trigeminal chemosensitivity across the adult life span. Perception \& Psychophysics, 65, 115-122.

Young, B. D. (2016). Smelling matter. Philosophical Psychology, 29(4), 520-534. 